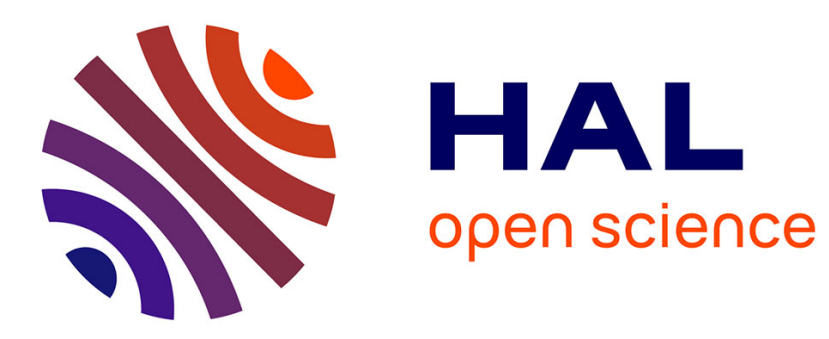

\title{
Shape parametrization of bio-mechanical finite element models based on medical images
}

\author{
Nathan Lauzeral, Domenico Borzacchiello, Michael Kugler, Daniel George, \\ Yves Rémond, Alexandre Hostettler, Francisco Chinesta
}

\section{To cite this version:}

Nathan Lauzeral, Domenico Borzacchiello, Michael Kugler, Daniel George, Yves Rémond, et al.. Shape parametrization of bio-mechanical finite element models based on medical images. Computer Methods in Biomechanics and Biomedical Engineering: Imaging \& Visualization, 2019, 7, pp.1 - 16. 10.1080/21681163.2018.1447400 . hal-01925458v3

\section{HAL Id: hal-01925458 \\ https://hal.science/hal-01925458v3}

Submitted on 20 Feb 2020

HAL is a multi-disciplinary open access archive for the deposit and dissemination of scientific research documents, whether they are published or not. The documents may come from teaching and research institutions in France or abroad, or from public or private research centers.
L'archive ouverte pluridisciplinaire HAL, est destinée au dépôt et à la diffusion de documents scientifiques de niveau recherche, publiés ou non, émanant des établissements d'enseignement et de recherche français ou étrangers, des laboratoires publics ou privés. 
To appear in Computer Methods in Biomechanics and Biomedical Engineering

Vol. 00, No. 00, Month 20XX, 1-16

\title{
Shape parametrization of bio-mechanical finite element models based on medical images
}

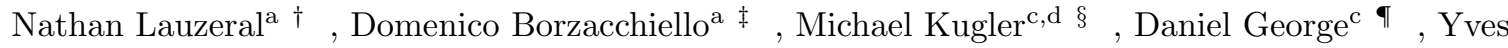 \\ Rémond $^{\mathrm{c}} \|$, Alexandre Hostettler ${ }^{\mathrm{d} \dagger \dagger}$, Francisco Chinesta ${ }^{\mathrm{b}} \ddagger$ \\ ${ }^{a}$ High Performance Computing Institute, EA7471, Ecole Centrale de Nantes, France; ${ }^{b}$ PIMM, ENSAM \\ ParisTech, CNRS, France; ${ }^{c}$ iCube, Université de Strasbourg, CNRS, France; ${ }^{d}$ IRCAD, France;
}

(Received 00 Month 20XX; accepted 00 Month 20XX)

\begin{abstract}
The main objective of this study is to combine the statistical shape analysis (SSA) with a morphing procedure in order to generate shape-parametric finite element models of tissues and organs and to explore the reliability and the limitations of this approach when applied to databases of real medical images. As classical statistical shape models are not always adapted to the morphing procedure, a new registration method was developed in order to maximize the morphing efficiency. The method was compared to the traditional iterative thin plate spline (iTPS). Two data sets of 33 proximal femora shapes and 385 liver shapes were used for the comparison. The principal component analysis (PCA) was used to get the principal morphing modes.

In terms of anatomical shape reconstruction (evaluated through the criteria of generalization, compactness, and specificity), our approach compared fairly well to the iTPS method, while performing remarkably better in terms of mesh quality, since it was less prone to generate invalid meshes in the interior. This was particularly true in the liver case. Such methodology offers a potential application for the generation of automated finite element (FE) models from medical images. Parametrized anatomical models can also be used to assess the influence of inter-patient variability on the biomechanical response of the tissues. Indeed, thanks to the shape parametrization the user would easily have access to a valid FE model for any shape belonging to the parameters subspace.
\end{abstract}

Keywords: statistical shape model, mesh morphing, data-driven modeling, patient-specific modeling, computational anatomy, liver atlas

\section{Introduction}

The FE method is widely used to simulate the physical behavior of problems with complex geometries. When the FE model is built upon real images the pre-processing setup can be time-consuming, especially in 3D and when the task has to be repeated several times for similar applications. This step often requires a manual intervention to assign boundary conditions or material properties. A lot of effort has been focused on the development of automated procedures for model generation from real data in order to avoid these overhead costs. A promising technique is the mesh warping (Couteau et al. 2000; Castellano-Smith et al. 2001; Sigal et al. 2008; Grosland et al. 2009; Bucki et al. 2010). The underlying idea is to define a template mesh that can be morphed in order to

\footnotetext{
$\dagger$ Corresponding author. Address: 1 rue de la Noë, 44300, Nantes, France. Email: nathan.lauzeral@ec-nantes.fr

¥Address: 1 rue de la Noë, 44300, Nantes, France. Email: domenico.borzacchiello@ec-nantes.fr

§Address: 300 Bd Sébastien Brant, 67400, Illkirch-Graffenstaden, France. Email: michael.kugler@unistra.fr

ฯAddress: 300 Bd Sébastien Brant, 67400, Illkirch-Graffenstaden, France. Email: george@unistra.fr

॥ Address: 300 Bd Sébastien Brant, 67400, Illkirch-Graffenstaden, France. Email: remond@unistra.fr

††Address: 1 Place de l'Hôpital, 67000, Strasbourg, France. Email: alexandre.hostettler@ircad.fr

¥¥Address: 151 Boulevard de l'Hôpital, 75013, Paris, France. Email: Francisco.Chinesta@ec-nantes.fr
} 
fit the target shape while keeping a good mesh quality. The procedure is often applied in a medical framework, the target shapes are then obtained from computed tomography (CT) scans or magnetic resonance images (MRI). A popular way of extracting the shape of the organs from such images is to use statistical shape models (SSMs) (Heimann and Meinzer 2009). SSMs are statistical representations of the organ shapes built from a training data set. A mesh is chosen as the reference shape and modes of variations of the geometry are associated with it, it allows to represent any shape similar to the ones of the training set.

The aim of this study is to fill the existing gap and bridge these two methodologies in order to automatically and quickly build volumetric meshes from SSMs. These latter are used to create a data-based shape parametrization allowing personalized FE modeling based on medical images. Moreover, shape parametric FE models are useful to quantify the trends of the shapes in the population and assess the influence of anatomy on biomechanical behavior. Indeed, from the parametrization it is possible to create any FE model of the same family while sharing a unique mesh connectivity and topology. This would allow propagating any anatomical landmark from the template to any generated shape. For the study of inter-population mechanical behavior variability, it would allow specifying only once the boundary conditions and material properties and to use them for any shape of generated FE model.

In recent years several studies have focused on the statistical representation of the whole body ( $\mathrm{Hu}$ 2018; Zhang et al. 2017; Beillas and Berthet 2017), also using morphing methods to generate complete FE models. In these studies the SSA is done on specific elements of the skeleton - i.e., the ribs (Weaver et al. 2014; Wang et al. 2016) - and particular points on the external body surface. Landmark-based morphing methods are then applied using radial basis functions such as the thin plate spline (TPS). Nonetheless, such kind of morphing has no consideration for the quality of the morphed mesh. To take into account such a factor, the method presented in this paper works in two steps: a SSA followed by a morphing procedure called iFEMWARP (Shontz and Vavasis 2012). In order to maximize the efficiency of the morphing step a new registration procedure, called thin plate spline-parametrized registration, is set up to create the SSMs. This new registration method is compared to the well-known iTPS in order to assess its relevance. The training sets used for the comparison contain 385 liver shapes and 33 proximal femora shapes. These databases contain 3D geometries of pre-processed medical images, hence the paper will not deal with image processing or segmentation.

\section{Materials and methods}

In this section a cloud of $3 \mathrm{D}$ points $p_{i}=\left(x_{i}, y_{i}, z_{i}\right) \in \mathrm{R}^{3}$, with $i=1, \ldots, v$, will be represented by the vector $\boldsymbol{X}=\left\{x_{1}, \ldots, x_{v}, y_{1}, \ldots, y_{v}, z_{1}, \ldots, z_{v}\right\}^{T} \in \mathrm{R}^{v \times 3}$. The Euclidean distance will be denoted by $\|\cdot\|$.

\section{$2.1 \quad$ Data}

The method was applied to two sets of 3D training shapes provided in $v t k$ format. In order to create the SSMs, a template shape was chosen for each one of the training sets. A first database of $n_{T}=385$ liver shapes was provided by the IRCAD (Institut de Recherche contre les Cancers de l'Appareil Digestif, Strasbourg, France). This database contains a large number of liver shapes collected from CT-scans or MRIs over several years, on patients in supine position. The stored meshes quality and precision evolved with the medical images segmentation methods. First, the different organs were identified in every single image independently, leading to discontinuities. This technique was completed with context-based treatment (Malandain et al. 1993) and topological identification (Dokládal et al. 1999) which improved greatly the organs consistency and shape. Additional post-treatments (Boltcheva et al. 2007) were done to guarantee manifoldness and nonovercrossing faces, which are compulsory for 3D-meshing. The integration of additional techniques 
like context base-voting (Chi et al. 2011) and region-growing algorithm (Frericks et al. 2004) lead to consequent improvement of the mesh precision, as well as a reduction of the error induced by the segmentation technician. As a result, the liver geometries used present a remarkable diversity in terms of source (male or female, healthy or unhealthy, ...) or in terms of method used for their generation. They present in particular a strong variation concerning their number of vertices, going from $v=2000$ to $v=150,000$, in order to describe a single liver surface. The liver template surface was described by $v=1393$ vertices and 2782 triangles (see Figure 1(a)). The volume was meshed with 10, 163 tetrahedra for a total of $v=2452$ vertices. Its maximum length was around $260 \mathrm{~mm}$. The second database contains $n_{T}=33$ proximal femora shapes freely provided by HazratiMarangalou (2013). The data were collected on 33 cadavers, 16 males and 17 females, between 61 and 95 years. The proximal femora template surface was described by $v=1384$ vertices and 2764 triangles (see Figure 1(b)). The volume was meshed with 8724 tetrahedra for a total of $v=2222$ vertices. Its maximum length was around $90 \mathrm{~mm}$.

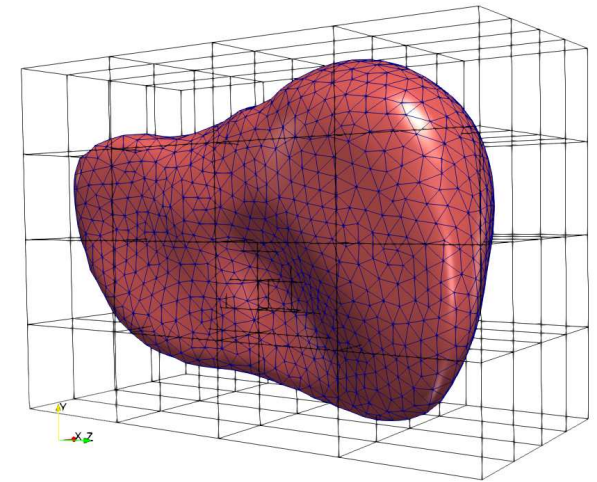

(a) Liver $\left(n_{b}=5\right)$

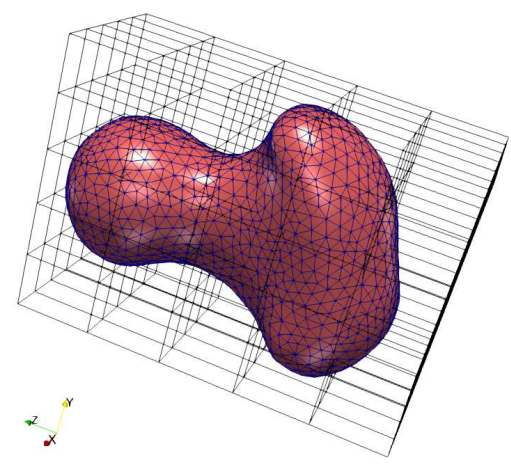

(b) Proximal femora $\left(n_{b}=6\right)$

Figure 1. Template meshes with their bounding boxes. The visualization was done on Paraview

\subsection{Creation of the FE model from SSMs}

The method presented in this article can be split into two parts: an offline stage where the SSMs are built and an online one where the FE model is reconstructed from the shape parametrization.

\section{Offline stage: construction of the SSMs}

The SSA presented here belongs to the point distribution model (PDM) family. Two major steps can be identified to build PDMs: the alignment of the shapes and the dimensionality reduction.

a. The alignment step works as follows: a template shape $S_{0}$ - a shape is described by a point cloud - is chosen. Each shape from the training set, subsequently mentioned as target, is rigidly registered onto the template. Then, the template is non-rigidly registered onto each target. For each non-rigid registration a deformation field corresponding to the template displacement is associated.

b. A dimensionality reduction method, most of the time the PCA (Jolliffe 2002), is then applied to this deformation database in order to find the principal modes of deformation associated with the template. By selecting the set of modes that best describes the observed variation the user is able to statistically describe any shape with a restricted number of modes. Any new shape $\tilde{\boldsymbol{X}}$ can be written as the sum of the mean shape $\overline{\boldsymbol{X}}$ 
and a linear combination of the selected principal modes $\phi_{i}$ :

$$
\tilde{\boldsymbol{X}}=\overline{\boldsymbol{X}}+\sum_{i=1}^{n} \xi_{i} \boldsymbol{\phi}_{i}
$$

where $\xi_{i}$ are the shape parameters. Finally, the subspace containing the shape parameters is defined.

2. Online stage: reconstruction of the FE model from the SSMs

a. First, the volumetric mesh of the template shape $S_{0}$ is computed.

b. For a given new shape the corresponding shape parameters from Equation 1 are identified. Consequently, the shape is now described by the same mesh connectivity and topology as the template.

c. As the template volumetric mesh is known the morphing method iFEMWARP can be applied to the new shape in order to create the FE model.

For each new shape the steps b. and c. have to be repeated.

In the remainder of the section we detail each step of the method.

\subsection{Offline training}

\subsubsection{Template shape}

The SSMs are built a first time by arbitrarily choosing a template shape. The mean geometry of all models under consideration is then chosen as the new template shape. Both surface and volume are meshed with gmsh (Geuzaine and Remacle 2009) using triangles and tetrahedra, respectively. Special attention is paid to the mesh's quality.

\subsubsection{Rigid registration}

All the positions, orientations and sizes between the targets and the template are minimized in order to keep only the shape variation. Two rigid registration methods are used sequentially. A Procrustes superimposition is applied through the principal axis transformation (PAT) method (Alpert et al. 1990; Maurer and Fitzpatrick 1993) followed by 30 iterations of the iterative closest point (ICP) (Besl et al. 1992; Chen and Medioni 1992) using point to point minimization (Kjer and Wilm 2010). The ICP alone is not sufficient because it tends to converge to local minima. The PAT allows doing a first basic registration taking into account the global shapes.

\subsubsection{Non-rigid registration}

The main idea to perform this registration is to minimize a functional

$$
I(\boldsymbol{u})=I_{m a t c h}(\boldsymbol{u})+I_{t p s}(\boldsymbol{u})
$$

through the use of a semi-implicit iterative algorithm using gradient descent. The functional is the sum of two terms: a point matching term $I_{\text {match }}$ and the shape bending energy $I_{\text {tps }}$ through a TPS parametrization. The target shape will be mentioned by $T$ and the corresponding deformed template shape by $S$. The initial template shape stays $S_{0}$. The non-rigid registration method will be called TPS-parametrized registration (TPS-PR).

2.3.3.1 Point matching term. The correspondence is usually performed to associate each node of the template with a pair among the target nodes. In this study a new correspondence method is used taking into account the nearest point in $T$ from each point $s$ in $S$ but also the nearest point 
in $S$ from each point $\boldsymbol{t}$ in $T$. For each point $\boldsymbol{s}_{0}$ in $S_{0}$ and $s$ its position in the deformed state, a displacement vector toward $T$ is defined as:

$$
\operatorname{Dist}\left(\boldsymbol{s}, \boldsymbol{s}_{0}, T\right)=\frac{1}{r+1}\left(d_{1}\left(\boldsymbol{s}, \boldsymbol{s}_{0}, T\right)+d_{2}\left(\boldsymbol{s}, \boldsymbol{s}_{0}, T\right)\right)
$$

where

$$
d_{1}:\left(\boldsymbol{s}, \boldsymbol{s}_{0}, T\right) \rightarrow \boldsymbol{t}-\boldsymbol{s}_{0} \quad / \quad \boldsymbol{t}=\underset{\boldsymbol{t} \in T}{\arg \min }(\|\boldsymbol{t}-\boldsymbol{s}\|)
$$

and

$$
d_{2}:\left(s, s_{0}, T\right) \rightarrow \sum_{i=1}^{r}\left(\boldsymbol{t}_{i}-\boldsymbol{s}_{0}\right) \quad / \quad \boldsymbol{t}_{i} \text { has } \boldsymbol{s} \text { as closest point }
$$

where $r \in \mathrm{N}$ is the number of points $\boldsymbol{t}_{i} \in T$ having $\boldsymbol{s}$ as the closest point. With this definition of distance between a point and a shape all the points of the target have an influence on the registration. For complete shape registration it allows avoiding to have non-registered parts. The point matching term between the template and a target is written:

$$
I_{\text {match }}(\boldsymbol{u})=\frac{1}{2}\|\boldsymbol{u}-\boldsymbol{d}\|^{2}
$$

where $\boldsymbol{u}$ is the unknown displacement associated with the template shape and $\boldsymbol{d}=\operatorname{Dist}\left(S, S_{0}, T\right)$.

2.3.3.2 Thin plate spline parametrization. The TPS is an efficient tool for shape matching and object recognition (Belongie et al. 2002), especially for biological applications (Bookstein 1989). The fundamental idea here is to parametrize the displacement of each point belonging to the template shape using a spline function $f$ :

$$
\boldsymbol{u}_{i}=f\left(x_{i}, y_{i}, z_{i} ; \boldsymbol{a}\right)
$$

where the parameters $\boldsymbol{a}$ are associated with the control points of a discrete bounding box like the ones shown in Figures 1(a) and 1(b). Requiring the smoothness of the registration is equivalent to minimize the "bending energy" of this bounding box. This reads as:

$I_{f}=\iiint_{\Omega}\left(\left(\frac{\partial^{2} f}{\partial x^{2}}\right)^{2}+\left(\frac{\partial^{2} f}{\partial y^{2}}\right)^{2}+\left(\frac{\partial^{2} f}{\partial z^{2}}\right)^{2}+2\left(\left(\frac{\partial^{2} f}{\partial x \partial y}\right)^{2}+\left(\frac{\partial^{2} f}{\partial x \partial z}\right)^{2}+\left(\frac{\partial^{2} f}{\partial y \partial z}\right)^{2}\right)\right) d x d y d z$

where $f$ is called the TPS interpolant. In practice Equations 5 and 6 can be easily evaluated through a FE approach considering Hermitian shape functions of order 2. In this case the bending energy is expressed as:

$$
I_{t p s}(\boldsymbol{a})=\frac{1}{2} \boldsymbol{a}^{T} \mathbf{L} \boldsymbol{a}
$$

where $\mathbf{L}$ is the discrete biharmonic operator obtained through traditional FE discretization techniques, while the displacements of the points are expressed by the linear mapping:

$$
\boldsymbol{u}=\mathrm{A} \boldsymbol{a}
$$

where $\mathbf{A}$ is the interpolation operator. The bounding box is fixed to $+5 \%$ the size of the template and the number of control points along each dimension $n_{b}$ can be freely chosen. By introducing Equations 4, 7 and 8 in Equation 2 we get the following expression:

$$
I(\boldsymbol{a})=\frac{1}{2}\|\mathbf{A} \boldsymbol{a}-\boldsymbol{d}\| \|^{2}+\frac{\lambda}{2} \boldsymbol{a}^{T} \mathbf{L} \boldsymbol{a}
$$


where $\lambda \in \mathrm{R}$ is a weighting scalar.

2.3.3.3 Resolution through gradient descent. As $I_{\text {match }}$ non linearly depends on $\boldsymbol{a}$, a non-linear semi-implicit scheme is used through gradient descent using the golden-section search. As the optimal value for the coefficient $\lambda$ can be hard to find manually, a new factor $\lambda_{r}$ is introduced as:

$$
\lambda=\lambda_{r} \cdot \frac{\max _{\alpha \in[0,1]} I_{\text {match }}(\boldsymbol{a})}{\max _{\alpha \in[0,1]} I_{\text {tps }}(\boldsymbol{a})} \quad \lambda_{r} \in \mathrm{R}^{+}
$$

where $\alpha$ is the golden-section search step. $\lambda_{r}$ represents the ratio between the maximum matching energy and the maximum bending energy. A high value $(>1)$ means the shape deformation will be highly penalized, a low value $(<1)$ means larger deformations are allowed at each increment. Once $\alpha$ is computed, the gradient descent step at iteration $k$ can be written as:

$$
\boldsymbol{a}^{(k+1)}-\boldsymbol{a}^{(k)}=-\alpha \frac{d I}{d \boldsymbol{a}}=-\alpha\left[\left(\lambda \mathbf{L}+\mathbf{A}^{T} \mathbf{A}\right) \boldsymbol{a}^{(k+1)}-\mathbf{A}^{T} \boldsymbol{d}^{(k)}\right] .
$$

Each time the golden-section search is run the value of $\lambda$ is actualized. In practice Equation 11 is solved iteratively until convergence. Each iteration involves the solution of a $12 n_{b}^{3} \times 12 n_{b}^{3}$ linear system. The factor 12 is coming from each control point having 12 degrees of freedom and the exponent 3 from the $3 \mathrm{D}$.

\subsubsection{Principal component analysis}

Once all shapes are non rigidly registered a displacement $\boldsymbol{a}_{i}$ is associated with each target $i$. The PCA is applied to the matrix $\left[\boldsymbol{a}_{1}, \ldots, \boldsymbol{a}_{n_{T}}\right]$. The principal modes $\boldsymbol{\phi}_{i}^{b}$ of the PCA associated with the $n$ largest eigenvalues $\lambda_{i}$ are chosen to form a reduced basis for $\boldsymbol{a}$. The size $n \ll 12 n_{b}^{3}$ of the basis is chosen depending on the percentage of variability $\lambda_{i} / \sum_{i=1}^{n_{T}} \lambda_{i}$ associated with each deformation mode. The representation of $\boldsymbol{a}$ in the reduced basis reads as:

$$
\tilde{\boldsymbol{a}}=\sum_{i=1}^{n} \xi_{i}^{b} \boldsymbol{\phi}_{i}^{b}
$$

where $\xi_{i}^{b}$ are the associated shape parameters. Thanks to Equation 8 a shape parametrization $\tilde{\boldsymbol{X}}$ can be written as:

$$
\tilde{\boldsymbol{X}}=\overline{\boldsymbol{X}}+\sum_{i=1}^{n} \xi_{i}^{b} \mathbf{A} \boldsymbol{\phi}_{i}^{b}
$$

\subsubsection{Statistical boundary shape models}

Each one of the shapes in the database can be described by a linear combination of shape parameters and principal modes (see Equation 13). But all combinations of modes cannot be considered as valid shapes. In order to avoid incoherent shapes, shape parameters ranges have to be bounded. The term " $\kappa$-boundary models" was introduced in Lu and Untaroiu (2013) to define the boundary models which cover $\kappa \%$ of the overall population. Various studies on anatomical elements as liver (Lu et al. 2013; Lu and Untaroiu 2014), clavicular cortical bone (Lu and Untaroiu 2013) or lunate and scaphoid bones (van de Giessen et al. 2010) have shown that the shape parameters tend to follow normal distributions over the training data set. Consequently, the shape space $[-3 \times \mathrm{SD}, 3 \times \mathrm{SD}]$ (SD stands for standard distribution) of each mode will define a 99.7\%-boundary model. A $q$ hyper-rectangle (Lu and Untaroiu 2013) is defined around the mean model, where $q$ is the number 
of selected modes. It describes a subspace in dimension $q$ where lies the shape parameters able to represent $99.7 \%$ of the whole population shape variations. The distribution normality is tested using a Kolmogorov-Smirnov test at the $5 \%$ significance level.

\subsection{Online stage}

\subsubsection{Shape identification from an out-of-sample geometry}

Once the offline stage is done, any new shape can be represented by Equation 13. The best way to register a new shape is to introduce Equation 12 into Equation 9 and minimize the function with respect to the shape parameters $\xi_{i}$. Indeed, the gradient descent step from Equation 11 becomes:

$$
\boldsymbol{\xi}^{(k+1)}-\boldsymbol{\xi}^{(k)}=-\alpha \frac{d I}{d \boldsymbol{\xi}}=-\alpha\left[\left(\lambda \boldsymbol{\Phi}^{T} \mathbf{L} \boldsymbol{\Phi}+\boldsymbol{\Phi}^{T} \mathbf{A}^{T} \mathbf{A} \boldsymbol{\Phi}\right) \boldsymbol{\xi}^{(k+1)}-\boldsymbol{\Phi}^{T} \mathbf{A}^{T} \boldsymbol{d}^{(k)}\right] .
$$

The computational cost is now reduced to the solution of a $n \times n$ system with $n \ll 12 n_{b}^{3}$. The most time-consuming part is the computation of $\boldsymbol{d}$, the correspondence vector. To simplify the calculations it is possible to take $\lambda$ constant. As the shape already belongs to a predefined subspace it will keep a coherent deformation. Besides, this allows storing the whole matrix $\lambda \boldsymbol{\Phi}^{T} \mathbf{L} \boldsymbol{\Phi}+\boldsymbol{\Phi}^{T} \mathbf{A}^{T} \mathbf{A} \boldsymbol{\Phi}$ for faster computation.

\subsubsection{Iterative FEMWARP}

The iFEMWARP was introduced in Shontz and Vavasis (2012) and is an extension of the FEMWARP (Baker 2002; Shontz and Vavasis 2010) which is a part of the linear weighted Laplacian smoothing framework (Shontz and Vavasis 2003). For a given mesh in 2D or 3D the FEMWARP assumes that the displacements of the boundary nodes are known. By considering a linear elastic deformation the position of the inner nodes can be easily computed by solving a linear system. Sometimes this procedure is not sufficient to untangle the mesh. A tangled mesh being a mesh where at least one of its elements has a null or negative Jacobian. The process is then iterated. At each iteration the Jacobians of all the elements are computed and those with a null or negative Jacobian have their rigidity $E$ increased by $50 \%$. The process is iterated until all elements have a strictly positive Jacobian. This method has been proved to be robust (Shontz and Vavasis 2012) and is quite easily implementable. A second parameter $\nu$ corresponding to the Poisson ratio also needs to be defined. To go further, we propose to enhance the method by taking into account the mesh quality. This latter is evaluated through its aspect ratio (AR) defined for a given tetrahedron as three times the quotient of inscribed and circumscribed sphere radii. It reads:

$$
A R=3 \frac{I R}{C R}
$$

where $I R$ (resp. $C R$ ) is the inscribed (resp. circumscribed) radius. A value of 1 means this is a regular tetrahedron, a value of 0 means the element is flat, also called silver. This metric is commonly used in the literature (Alliez et al. 2005) or in mesh generator software such as gmsh. To run FE computations, it is often advised that the majority of elements has an AR superior to 0.3. However, there is no theoretical back-up proving this threshold value. To improve the iFEMWARP, instead of only increasing the rigidity of elements with a negative Jacobian, the rigidity of poor quality elements is also increased at each increment following:

$$
E_{e}^{i+1}=E_{e}^{i}\left(1+0.5\left(1-\frac{A R^{i}}{0.3}\right)^{2}\right)
$$


where $E_{e}^{i}$ is the Young modulus of the $e$-th element at increment $i$ and $A R^{i}$ its aspect ratio. In our case the displacements of the boundary nodes are defined by the shape parametrization. Once the shape parameters are identified the morphing can be directly applied. In this paper the iFEMWARP was used with $E=1 k P a$ and $\nu=0.4$. These two parameters are related to the morphing procedure and are called after the elastic constants due to the similarity of the morphing functional with the elastic energy. Nonetheless, these are not related to the tissue's real mechanical properties. All elements start with the same rigidity at the first iteration, after which their relative stiffness is updated. The parameter $\nu$ is chosen so as to limit the compressibility to avoid distorting too much good quality elements during the iterations.

\subsection{Validation}

For completeness we review the iTPS method technique that we used as a reference to assess the quality of our results.

\subsubsection{Iterative thin plate spline method}

The rigid registration was done using the same method as for the TPS-PR. For the correspondence, landmarks were placed on the template shape by splitting the space into equal-sized cubic grid cells. For each cell containing a surface point a landmark was created by taking the closest point to the cell's center. Here the space was refined into cells of $7 \times 7 \times 7 \mathrm{~mm}^{3}$ resulting in around 1300 template landmarks for the proximal femora and the liver. Each landmark was associated with its corresponding point using the "Giessen" approach (Van De Giessen et al. 2009). This method was proven more efficient than using only the Euclidean distance (Lu and Untaroiu 2014). Once the initial correspondence was done the registration was computed by minimizing the energy:

$$
I(f)=\sum_{i=1}^{n}\left\|\boldsymbol{t}_{i}-f\left(\boldsymbol{s}_{i}\right)\right\|+\lambda I_{f}
$$

where $\boldsymbol{s}_{\boldsymbol{i}}$ are the template landmarks and $\boldsymbol{t}_{i}$ their corresponding points on the target. $\lambda$ is the regularization parameter and $I_{f}$ is defined in Equation 6. The interpolant $f$ has the form:

$$
f(x, y, z)=a_{1}+a_{2} x+a_{3} y+a_{4} z+\sum_{i=1}^{n} w_{i} U\left(\left\|s_{i}-(x, y, z)\right\|\right)
$$

where $U(r)=a b s(r)$ is a radial basis function.

By iterating the matching and registration processes the final registration is improved. In this paper $\lambda=\alpha^{2} \lambda_{0}$ where $\lambda_{0}=100$ and $\alpha$ is the mean edge length between two points in the set, as recommended in Belongie et al. (2002). The maximum number of iterations was fixed to 35 .

\subsubsection{Error metrics}

The quality of each registration method was evaluated using three metrics introduced in Davies (2002): the compactness, the generalization, and the specificity. They depend on $M$, the number of modes of variation used for the reconstruction. The measure of the compactness is defined by:

$$
C(M)=\sum_{m=1}^{M} \lambda^{m}
$$

where $\lambda^{m}$ is the $m$-th eigenvalue calculated from PCA. A compact model is one with small variance and which requires a small set of parameters to describe the shape. Then, the measure of the 
generalization is defined by:

$$
G(M)=\frac{1}{n_{T}} \sum_{i=1}^{n_{T}} \operatorname{Dist}\left(S_{i}^{0}, S_{i}(M)\right)
$$

where $n_{T}$ is the number of shapes of the training set, $S_{i}^{0}$ the $i$-th registered shape and $S_{i}$ the corresponding reconstructed shape. The generalization is the ability of the SSMs to represent a shape out of the training set. To evaluate it the SSM was built using $n_{T}-1$ shape and the last shape was then reconstructed using $M$ modes. The distance between the reconstruction and the ground truth was then evaluated. This leave-one-out test was repeated for all the shapes in the database and then averaged. Finally, the measure of the specificity is defined by:

$$
S(M)=\frac{1}{N} \sum_{i=1}^{N} \min _{T_{j} \in \mathcal{T}_{S}} \operatorname{Dist}\left(T_{j}, S_{i}(M)\right)
$$

where $N$ is the number of generated shapes, $S_{i}$ is a randomly generated shape and $\mathcal{T}_{S}$ the training set. The specificity is the ability to only represent shapes that are similar to the training set ones. A new set of 1000 uniformly distributed shapes was generated from the SSMs for $M$ modes. For each generated shape the distance was computed from its closest shape from the training set. The mean value of the distances was used as the measure of the specificity.

The distance between two shapes was defined in Xu et al. (2013) as:

$$
\operatorname{Dist}(S, T)=\frac{1}{2}\left[\frac{1}{N_{s}} \sum_{\boldsymbol{s} \in S} \min _{\boldsymbol{t} \in T}\|\boldsymbol{s}-\boldsymbol{t}\|+\frac{1}{N_{t}} \sum_{\boldsymbol{t} \in T} \min _{\boldsymbol{s} \in S}\|\boldsymbol{s}-\boldsymbol{t}\|\right]
$$

where $\boldsymbol{s}$ (resp. $\boldsymbol{t}$ ) are the points belonging to $S$ (resp. T) and $N_{s}$ (resp. $N_{t}$ ) their number. The smaller the value, the more similar the shapes. For all of these metrics smaller values mean a better approach. As they depend on the training set, there is no threshold value allowing evaluating the quality of the registration method, that is why we compared the TPS-PR with the iTPS.

To assess the efficiency of the iFEMWARP to describe good volumetric meshes from the SSMs the regularity and quality of the meshes were evaluated. A finite element $e$ is said to be regular if its Jacobian is positive in all of his nodes. For a specified number of mode $M, 1000$ instances normally distributed were generated within the boundaries (Botev 2017) using the SSMs. The iFEMWARP was then applied to reconstruct their volumes. If the morphing did not succeed to untangle the mesh in less than 30 iterations the method was considered as a failure. The percentage of failure defined the regularity error $\mathrm{R}(M)$. SSMs built with the iTPS and the TPS-PR were compared. The mesh quality was also evaluated by generating 1000 normally distributed instances, but for the liver only and for a fixed number of mode $M=30$. Only the TPS-PR built SSMs were considered. For each generated instance the AR of the mesh was evaluated. The normal distribution of the parameters is justified by the fact that the targets' shape parameters are also normally distributed.

\section{Results}

For the liver (resp. the femur) the TPS-PR was run with $n_{b}=5$ (resp. $n_{b}=6$ ) (see Figure 1(a) and $1(\mathrm{~b}))$ and $\lambda_{r}=0.3 .35$ increments were done for each target.

As mentioned in Paragraph 2.3.5 the normality of the shape parameters had to be tested to validate the choice of the boundary shape model. In practice, when using the TPS-PR method only the $15^{\text {th }}$ liver mode and the first of the femur did not strictly follow a normal distribution (see Figures 
2(a) and 2(b)). The hypothesis of normal distributions was acceptable and the choice of the shape parameter subspace was coherent.

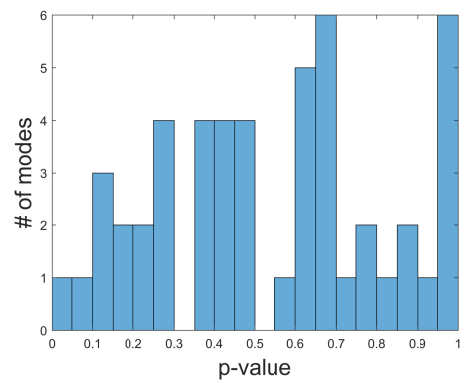

(a) Histogram of the p-values for the liver data, only the 50 first modes are represented.

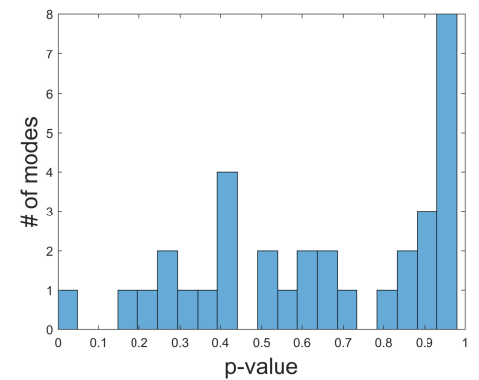

(b) Histogram of the p-values for the femur data, all 33 modes are represented.

Figure 2. Kolmogorov-Smirnov test at the $5 \%$ significance level applied to the shape parameters of the registered targets obtained with the TPS-PR. A $p$-value superior to 0.05 means the data distribution is not significantly different from the normal distribution.

For both the livers and the femurs the TPS-PR models were more compact (Figures 3(a) and 4(a)). This is due to the fact that the evaluation was done on the bounding box degrees of freedom which are fewer than the number of vertices. The generalization was slightly better for the liver database (Figure 3(b)) and almost equivalent for the femur one (Figure 4(b)). The specificity was worse for the liver but better for the femur (Figures 3(c) and 4(c)). Globally, for this set of parameters both methods behaved identically, the compactness put apart.

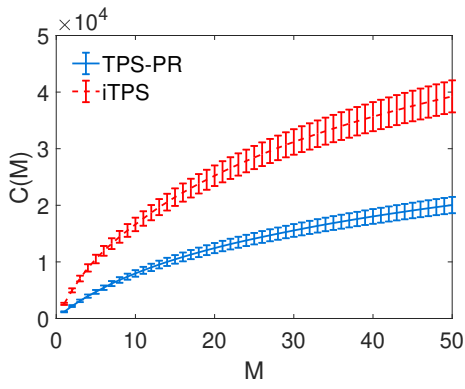

(a) Compactness

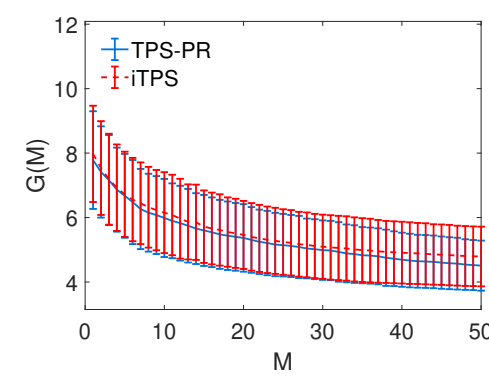

(b) Generalization

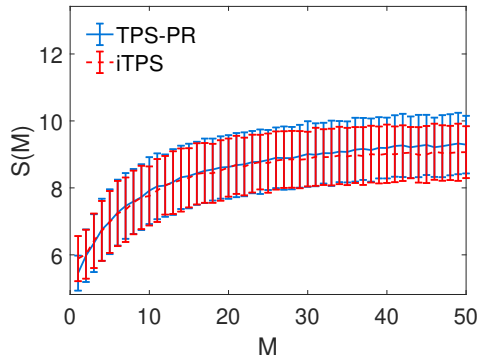

(c) Specificity

Figure 3. Comparison of the methods for the liver database (compactness, generalization, and specificity). For a given number of modes lower values mean a better method. The error bar is one standard deviation.

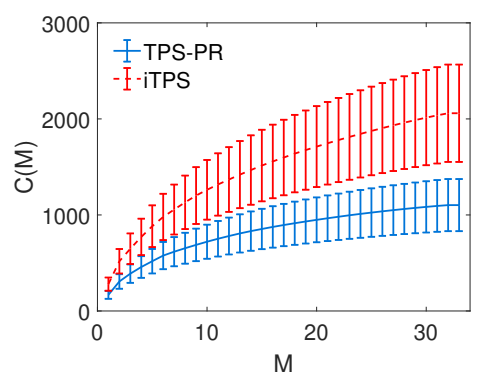

(a) Compactness

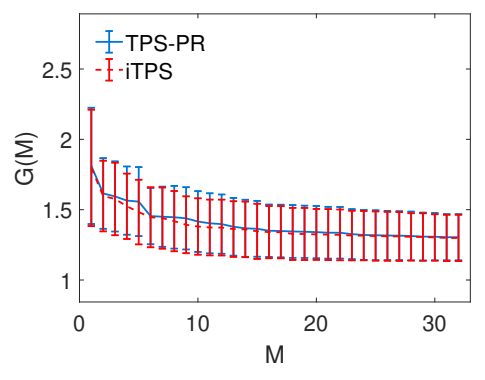

(b) Generalization

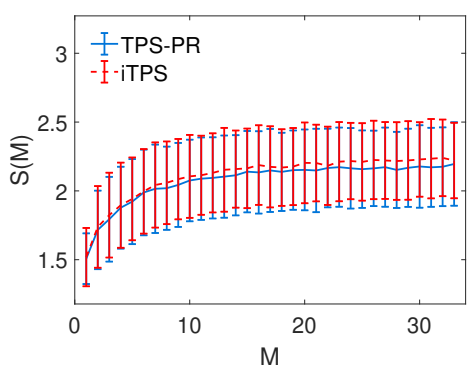

(c) Specificity

Figure 4. Comparison of the methods for the femur database (compactness, generalization, and specificity). For a given number of modes lower values mean a better method. The error bar is one standard deviation. 
The error done during the morphing of the liver instances is presented in Figure 5. For the femur no tangled meshes were produced with both methods. This was most likely caused by the fact that bones have small shape variations (see Table 1). Hence, the mesh was not under large deformations during registration and kept its integrity. In the case of the liver the TPS-PR produced few tangled meshes starting from $M=16$, but always less than $1 \%$ of the generated shapes. On the other hand, the iTPS produced tangled meshes starting from $M=2$ and the percentage of irregular meshes steadily increased until $8 \%$ for $M=50$. Globally, the error was bigger in the liver case because the database is larger and there is more variability in its anatomy (see Table 2). Moreover, with a large database, the probability to encounter difficult shapes to register increases and poorly meshed surfaces are introduced.

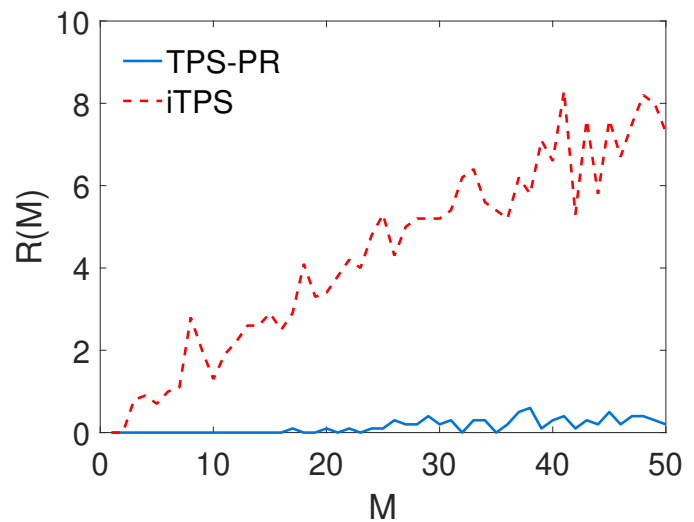

Figure 5. Percentage of tangled meshes among the 1000 liver instances generated for each mode $M$ after 30 iterations of the iFEMWARP.

The quality results are presented in Figure 6 for $M=30$ in the liver case and for 30 iterations of the iFEMWARP. The SSMs were built using the TPS-PR. In Figure 6(b) the mean AR over 1000 randomly generated shapes is presented in a histogram, showing the efficiency of the enhanced iFEMWARP and the good averaged quality of the morphed meshes. The quality distribution is slightly wider than the template one but stays within an acceptable range. An example of a generated mesh can be seen in Figure 6(a) with the value of its AR represented to testify of the good behavior of the method.

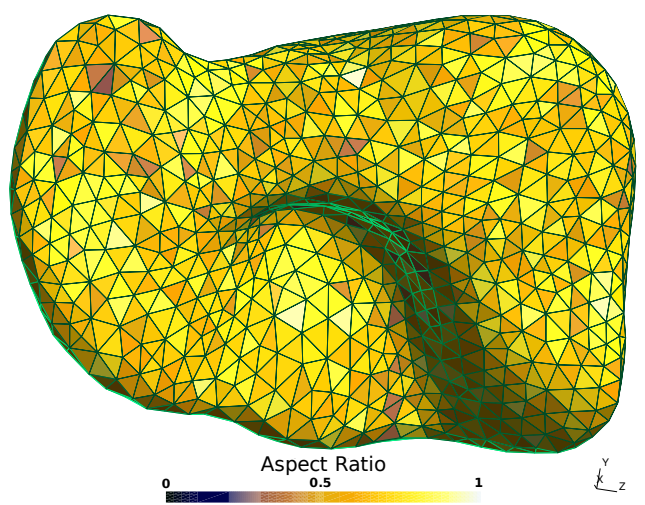

(a) AR map of a randomly generated liver FE model. The visualization is done on gmsh.

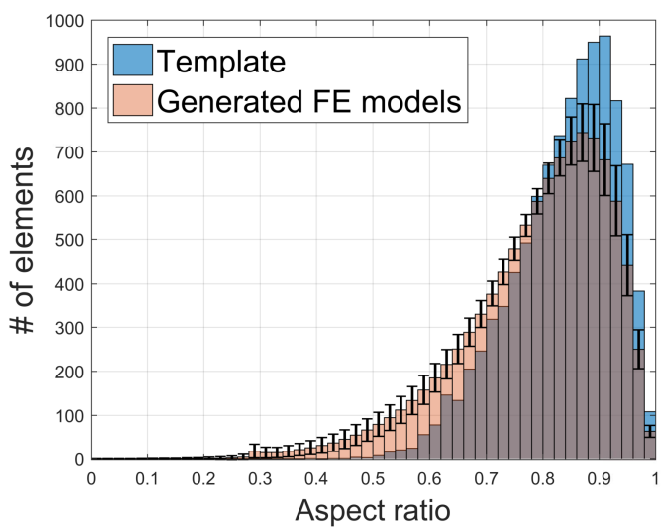

(b) Histogram of the mean AR over 1000 randomly generated shapes. The error bar corresponds to one standard deviation. The template AR histogram is also represented to provide a ground truth.

Figure 6. Application of the enhanced iFEMWARP on the liver SSMs for $M=30.30$ iFEMWARP iterations were done. 


\section{Discussion}

This study has been the opportunity to create SSMs from a large database of 385 liver shapes. This is to our knowledge one of the biggest liver training sets used in medical SSA coming from real data. With such a large database it is hard to describe the shape with a restricted number of parameters. Visually, at least 30 to 50 modes were required to describe all shapes with fidelity. The test that has been performed showed how a substantial difference arises when applying the method to real and large data sets and it would be interesting to see how it behaves toward others shapes databases. Indeed, as presented for the femur data set, the method works fine, regardless of the registration method. On the contrary, for the liver the regularity error was higher but the TPS-PR method succeeded to limit it under $1 \%$. We observed that the true benefits of using the TPS-PR were more evident when larger data sets were used or when the shapes presented a greater variability. Concerning the quality of the generated meshes, we showed that they are globally adapted to run FE simulations. However, the AR alone cannot be considered as an insurance of the good mesh quality. Some invalid meshes may still be generated.

The main limitation of the method is that the TPS-PR tends to smooth the target shape. Hence, if small details with strong variations need to be represented the method will probably not be able to fit them. The choice of the parameters has a strong influence on the results and sometimes the best option results from a tradeoff between the quality of surface registration and that of the volume mesh. The parameter $\lambda_{r}$ is mainly used as a way to "penalize" the registration and improve the correspondence. Besides, $n_{b}$ allows fostering either the quality of the registration for high values either the chances of success of the morphing for lower values. A small $n_{b}$ will smooth the registration and consequently preserve the mesh quality. Smoothing is also necessary in order to eliminate numerical artifacts from the FE solutions, which may arise from the presence of excessively distorted elements or sharp corners. The idea to use a bounding box to penalize the deformation and smooth the surface has already been tackled in Bucki et al. (2010) to register a generic volumetric mesh onto the patient organ shape, in conjunction with a mesh regularity and quality correction. This correction step presented in Bucki et al. (2011) could also be used in our case as a backup solution to untangle meshes.

Concerning the computational time, with a MATLAB (The MathWorks, Inc. USA) implementation the iFEMWARP took around 2 seconds per iteration for the proximal femoral and 3 seconds for the liver. Most of the time the convergence was reached in 1 iteration. The most difficult shapes to reconstruct were located on the limits of the boundary model but they also were statistically less likely to be observed. For the registration, an iteration of the TPS-PR took between 4 and 0.2 seconds depending if the golden-section search was used or not. It is possible to further accelerate the method by not enforcing the golden-section search at each iteration. Indeed, acceptable results can be obtained when the golden-section search is only performed every few iterations. On the other hand, it introduces a significant speed-up since this task is the most time-consuming.

\section{Conclusion}

We presented and tested a procedure to automatically generate FE models from medical images by coupling SSMs to a mesh morphing technique. A new registration method has been developed to build the SSMs in order to increase the efficiency of the morphing method. Concerning the quality of the reconstructed shapes we showed how the proposed TPS-PR performs equally well as other traditional techniques like iTPS while significantly improving the quality of interior volume meshing. The method can be in principle coupled to other more sophisticated registration techniques (e.g., the SLIDE method (Dalal et al. 2007) which has been proven to be really efficient). However, the choice of the particular approach adopted in this paper is justified by its flexibility and robustness when applied to a large and rich data set. This approach can be potentially very efficient 
in automated FE model generation from real data. Indeed, instead of registering a template mesh onto a specific target we create first a shape-parametrized model that allows us to apply a fast morphing algorithm afterward. By doing so the application is faster and enables the creation of shape-realistic FE models.

This method was developed with the intent of enabling efficient shape parametrization of FE models for parametric model order reduction (MOR). Our ongoing studies are focused on shape parametric MOR for patient-specific liver mechanics, based on the approach presented in Borzacchiello et al. (2017) and will be the object of upcoming work.

\section{Acknowledgments}

This work was performed by using HPC resources of the Centrale Nantes Supercomputing Centre on the cluster Liger, granted by the High Performance Computing Institute (ICI) (https://ici.ec-nantes.fr/).

This research was financially supported by BPI France through 3D-Surg, a project of the "Programme d'Investissements d'Avenir - PIA" of the French Government. (http://www.3d-surg.eu/). 
1181 18188 18145 मी 182 11528 


\section{References}

Alliez P, Cohen-Steiner D, Yvinec M, Desbrun M. 2005. Variational tetrahedral meshing. In: ACM Transactions on Graphics (TOG). ACM; p. 617-625. vol. 24.

Alpert N, Bradshaw J, Kennedy D, Correia J. 1990. The principal axes transformation - a method for image registration. Journal of nuclear medicine. 31(10):1717-1722.

Baker TJ. 2002. Mesh movement and metamorphosis. Engineering with Computers. 18(3):188-198.

Beillas P, Berthet F. 2017. An investigation of human body model morphing for the assessment of abdomen responses to impact against a population of test subjects. Traffic injury prevention. (just-accepted):00-00.

Belongie S, Malik J, Puzicha J. 2002. Shape matching and object recognition using shape contexts. IEEE transactions on pattern analysis and machine intelligence. 24(4):509-522.

Besl PJ, McKay ND, et al. 1992. A method for registration of 3-d shapes. IEEE Transactions on pattern analysis and machine intelligence. 14(2):239-256.

Boltcheva D, Bechmann D, Thery S. 2007. Discrete delaunay: Boundary extraction from voxel objects. In: 3-D Digital Imaging and Modeling, 2007. 3DIM'07. Sixth International Conference on. IEEE; p. $209-216$.

Bookstein FL. 1989. Principal warps: Thin-plate splines and the decomposition of deformations. IEEE Transactions on pattern analysis and machine intelligence. 11(6):567-585.

Borzacchiello D, Aguado JV, Chinesta F. 2017. Non-intrusive sparse subspace learning for parametrized problems. Archives of Computational Methods in Engineering:1-24.

Botev Z. 2017. The normal law under linear restrictions: simulation and estimation via minimax tilting. Journal of the Royal Statistical Society: Series B (Statistical Methodology). 79(1):125-148.

Bucki M, Lobos C, Payan Y. 2010. A fast and robust patient specific finite element mesh registration technique: application to 60 clinical cases. Medical image analysis. 14(3):303-317.

Bucki M, Lobos C, Payan Y, Hitschfeld N. 2011. Jacobian-based repair method for finite element meshes after registration. Engineering with Computers. 27(3):285-297.

Castellano-Smith AD, Hartkens T, Schnabel J, Hose DR, Liu H, Hall WA, Truwit CL, Hawkes DJ, Hill DL. 2001. Constructing patient specific models for correcting intraoperative brain deformation. In: International Conference on Medical Image Computing and Computer-Assisted Intervention. Springer; p. 10911098.

Chen Y, Medioni G. 1992. Object modelling by registration of multiple range images. Image and vision computing. 10(3):145-155.

Chi Y, Liu J, Venkatesh SK, Huang S, Zhou J, Tian Q, Nowinski WL. 2011. Segmentation of liver vasculature from contrast enhanced ct images using context-based voting. IEEE Transactions on Biomedical Engineering. 58(8):2144-2153.

Couteau B, Payan Y, Lavallée S. 2000. The mesh-matching algorithm: an automatic 3d mesh generator for finite element structures. Journal of biomechanics. 33(8):1005-1009.

Dalal P, Munsell BC, Wang S, Tang J, Oliver K, Ninomiya H, Zhou X, Fujita H. 2007. A fast 3d correspondence method for statistical shape modeling. In: Computer Vision and Pattern Recognition, 2007. CVPR'07. IEEE Conference on. IEEE; p. 1-8.

Davies RH. 2002. Learning shape: optimal models for analysing natural variability [dissertation].

Dokládal P, Lohou C, Perroton L, Bertrand G. 1999. Liver blood vessels extraction by a 3-d topological approach. In: Medical Image Computing and Computer-Assisted Intervention-MICCAI'99. Springer; p. 98-105.

Frericks BB, Caldarone FC, Nashan B, Savellano DH, Stamm G, Kirchhoff TD, Shin HO, Schenk A, Selle D, Spindler W, et al. 2004. 3d ct modeling of hepatic vessel architecture and volume calculation in living donated liver transplantation. European radiology. 14(2):326-333.

Geuzaine C, Remacle JF. 2009. Gmsh: A 3-d finite element mesh generator with built-in pre-and postprocessing facilities. International journal for numerical methods in engineering. 79(11):1309-1331.

Grosland NM, Bafna R, Magnotta VA. 2009. Automated hexahedral meshing of anatomic structures using deformable registration. Computer methods in biomechanics and biomedical engineering. 12(1):35-43.

Hazrati-Marangalou J. 2013. Database of femur samples. Available from: https://data.4tu.nl/ repository/uuid:4ae59365-92f0-480b-a899-ade34bc84a00.

Heimann T, Meinzer HP. 2009. Statistical shape models for 3d medical image segmentation: a review. Medical image analysis. 13(4):543-563.

Hu J. 2018. Parametric human modeling. In: Basic finite element method as applied to injury biomechanics. Elsevier; p. 417-445. 
Jolliffe IT. 2002. Principal component analysis, second ed. Springer.

Kjer HM, Wilm J. 2010. Evaluation of surface registration algorithms for pet motion correction [dissertation]. Technical University of Denmark, DTU, DK-2800 Kgs. Lyngby, Denmark.

Lu YC, Kemper AR, Gayzik S, Untaroiu CD, Beillas P. 2013. Statistical modeling of human liver incorporating the variations in shape, size, and material properties. Stapp car crash journal. 57:285-311.

Lu YC, Untaroiu CD. 2013. Statistical shape analysis of clavicular cortical bone with applications to the development of mean and boundary shape models. Computer methods and programs in biomedicine. 111(3):613-628.

Lu YC, Untaroiu CD. 2014. A statistical geometrical description of the human liver for probabilistic occupant models. Journal of biomechanics. 47(15):3681-3688.

Malandain G, Bertrand G, Ayache N. 1993. Topological segmentation of discrete surfaces. International journal of computer vision. 10(2):183-197.

Maurer CR, Fitzpatrick JM. 1993. A review of medical image registration. Interactive image-guided neurosurgery:17-44.

Shontz SM, Vavasis SA. 2003. A mesh warping algorithm based on weighted laplacian smoothing. In: Proceedings of 12th International Meshing Roundtable. p. 147-158.

Shontz SM, Vavasis SA. 2010. Analysis of and workarounds for element reversal for a finite element-based algorithm for warping triangular and tetrahedral meshes. BIT Numerical Mathematics. 50(4):863-884.

Shontz SM, Vavasis SA. 2012. A robust solution procedure for hyperelastic solids with large boundary deformation. Engineering with Computers. 28(2):135-147.

Sigal IA, Hardisty MR, Whyne CM. 2008. Mesh-morphing algorithms for specimen-specific finite element modeling. Journal of biomechanics. 41(7):1381-1389.

van de Giessen M, Foumani M, Streekstra GJ, Strackee SD, Maas M, van Vliet LJ, Grimbergen KA, Vos FM. 2010. Statistical descriptions of scaphoid and lunate bone shapes. Journal of biomechanics. 43(8):14631469.

Van De Giessen M, Smitsman N, Strackee SD, Van Vliet LJ, Grimbergen KA, Streekstra GJ, Vos FM. 2009. A statistical description of the articulating ulna surface for prosthesis design. In: Biomedical Imaging: From Nano to Macro, 2009. ISBI'09. IEEE International Symposium on. IEEE; p. 678-681.

Wang Y, Cao L, Bai Z, Reed MP, Rupp JD, Hoff CN, Hu J. 2016. A parametric ribcage geometry model accounting for variations among the adult population. Journal of biomechanics. 49(13):2791-2798.

Weaver AA, Schoell SL, Stitzel JD. 2014. Morphometric analysis of variation in the ribs with age and sex. Journal of anatomy. 225(2):246-261.

Xu R, Zhou X, Hirano Y, Tachibana R, Hara T, Kido S, Fujita H. 2013. Particle system based adaptive sampling on spherical parameter space to improve the mdl method for construction of statistical shape models. Computational and mathematical methods in medicine. 2013.

Zhang K, Cao L, Fanta A, Reed MP, Neal M, Wang JT, Lin CH, Hu J. 2017. An automated method to morph finite element whole-body human models with a wide range of stature and body shape for both men and women. Journal of biomechanics. 60:253-260. 